\title{
Los Hydropsychidae (Insecta: Trichoptera) del río Oja (La Rioja, España)
}

\author{
María Valladolid $^{1, *}$, Juan José Martínez-Bastida ${ }^{2}$ y Mercedes Arauzo ${ }^{2}$ \\ 1 Departamento de Biodiversidad y Biología Evolutiva, Museo Nacional de Ciencias Naturales (CSIC), c/ José \\ Gutiérrez Abascal, 2, 28006 Madrid, España. \\ 2 Departamento de Contaminación Ambiental, Centro de Ciencias Medioambientales, (CSIC), C/ Serrano 115 \\ dpdo.28006 Madrid, España. Martinez.Bastida@ccma.csic.es, mercedes@ccma.csic.es
}

* Autor responsable de la correspondencia: marval@mncn.csic.es

\section{RESUMEN}

\section{Los Hydropsychidae (Insecta: Trichoptera) del río Oja (La Rioja, España)}

Durante el período 2003-2005 se llevaron a cabo una serie de muestreos de macroinvertebrados en el río Oja (La Rioja, España), tanto cuantitativos (2003-2004), como cualitativos (2004-2005), en cinco puntos situados a lo largo del cauce principal y uno más en cabecera, incorporado en 2005. En cuanto a los Tricópteros encontrados, la familia Hydropsychidae es la dominante con 10 especies: Hydropsyche ambigua, H. angustipennis, H. bulbifera, H. dinarica, H. exocellata, H. incognita, H. cf. incognita, H. gr. instabilis, H. lobata y $H$. siltalai. La riqueza en especies es alta, tanto si se compara con estudios de ríos españoles (media de 6-7 especies por cauce) como si se hace con cuencas completas (media de 6-10 especies). Basándonos en la distribución de las especies se han definido tres zonas: ritron, con $H$. ambigua, $H$. lobata, H. dinarica y H. bulbifera, que comprendería desde la cabecera hasta el punto 1; potamon, con H. angustipennis, H. exocellata, H. incognita, H. cf. incognita, H. gr. instabilis y H. siltalai, que comprendería desde el punto 3 hasta el punto 5, y una zona intermedia, donde aparece una mezcla de especies del ritron y del potamon (H. bulbifera, $H$. dinarica, $H$. exocellata, $H$. gr. instabilis y $H$. siltalai), que se alternan según la época del año.

Palabras clave: Trichoptera, Hydropsychidae, río Oja.

\section{ABSTRACT}

\section{The Hydropsychidae (Insecta: Trichoptera) of the Oja river (La Rioja, Spain)}

During the 2003-2005 period, a series of macroinvertebrate samplings were carried out in the Oja river (La Rioja, Spain), both quantitative (2003-2004), and qualitative (2004-2005), in 5 sampling stations throughout the main course of the river and one more sampling station upstream, near headwaters, incorporated in 2005. In reference to the found Trichoptera, the Hydropsychidae is the dominant family with 10 species: Hydropsyche ambigua, $\mathrm{H}$. angustipennis, $\mathrm{H}$. bulbifera, $\mathrm{H}$. dinarica, H. exocellata, H. incognita, H. cf. incognita, H. gr. instabilis, H. lobata and H. siltalai. Species richness is high not only if we compare with data from other Spanish rivers (6-7 species in average) but also with data from studies of river basins (6-10 species in average). Based on species distribution, we could define three areas: rithron, with $\mathrm{H}$. ambigua, $\mathrm{H}$. lobata, $\mathrm{H}$. dinarica, and $\mathrm{H}$. bulbifera, from source to station 1; potamon, with $\mathrm{H}$. angustipennis, $\mathrm{H}$. exocellata, $\mathrm{H}$. incognita, $\mathrm{H}$. cf. incognita, H. gr. Instabilis, and $\mathrm{H}$. siltalai, from station 3 to station 5, and an intermediate zone, where we found a mixture of species of rithron and potamon ( $\mathrm{H}$. bulbifera, $\mathrm{H}$. dinarica, $\mathrm{H}$. exocellata, $\mathrm{H}$. gr. instabilis and $\mathrm{H}$. siltalai), which alternate throughout the year.

Keywords: Trichoptera, Hydropsychidae, Oja River. 


\section{INTRODUCCIÓN}

Los Hydropsychidae son una familia de Tricópteros bien diversificada a nivel mundial, que constituye un elemento frecuente y abundante en todo tipo de corrientes de agua, desde ríos pequeños y arroyos hasta grandes cauces (Verneaux \& Faessel 1976; Bournaud et al. 1982; García de Jalón 1986). En los ríos mediterráneos son una de las familias más importantes, tanto en abundancia como en riqueza específica (Giudicelli et al., 1985).

El estudio de las especies ibéricas hasta fechas recientes ha sido dificultoso, ya que, aunque había numerosas claves de especies europeas, estaban incompletas y no se ajustaban a las especies españolas. Publicaciones españolas sobre los Hydropsychidae son p.e. las de García de Jalón (1981, 1983) y Millet (1983) con descripción de larvas y una pequeña clave de algunas especies, y más recientemente, las de Zamora-Muñoz et al. (1995) y la de VieiraLanero (2000), la primera dedicada a las larvas de las especies conocidas hasta ese momento, incluyendo una clave, y la segunda sobre los estadios larvarios de las especies presentes en Galicia, también con clave para estas especies concretas. La lista se puede completar con descripciones de nuevas especies de la fauna ibérica, como p.e. González \& Malicky (1999); Vieira-Lanero et al. (2001) y Zamora-Muñoz et al. (2002). En cuanto a la faunística, en la literatura podemos encontrar tanto publicaciones dedicadas a los Hydropsychidae (Herranz \& García de Jalón 1984; González \& Botosaneanu 1985; García de Jalón 1986; Basaguren 1990) como referencias a esta familia en estudios generales de Tricópteros (García de Jalón 1982 a, b; Puig et al., 1984, 1987; Miranda Braga, 1987; Ruiz et al., 2001; Vieira-Lanero, 2002; Bonada et al., 2004).

En este estudio presentamos los primeros datos obtenidos sobre las especies de Hydropsychidae del río Oja, como parte de un trabajo más amplio sobre los Tricópteros de este río (de próxima publicación). Su importancia radica, por una parte, en que es la primera vez que se estudian las comunidades bentónicas de este río, y
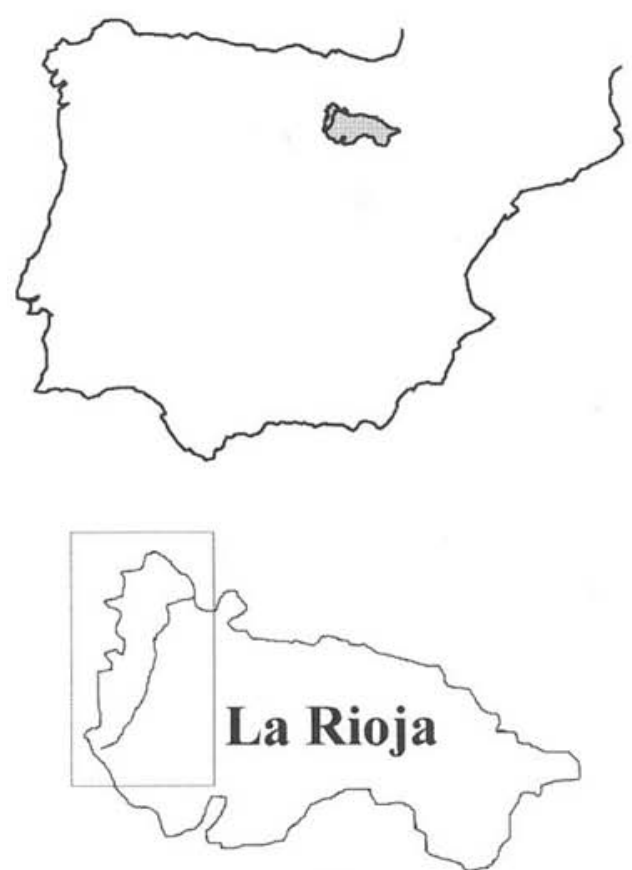

Ebro

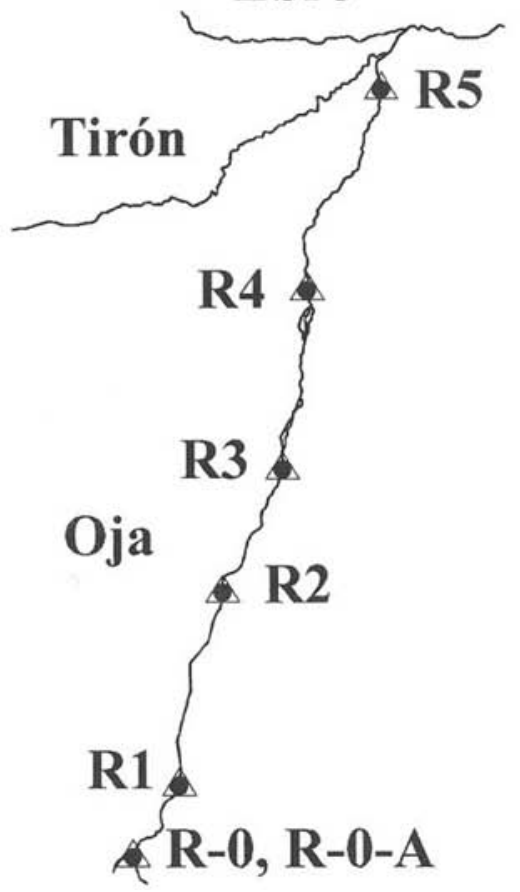

Figura 1. Localización de los puntos de muestreo. R0: Oja-Embalse del Águila, R-1: Oja-Arroyo Ortigal, R-2: Ezcaray, R-3: Santurde, R-4: Santo Domingo de la Calzada, R-5: Casalarreina. Location of sampling points. R-0: OjaEmbalse del Águila, R-1: Oja-Arroyo Ortigal, R-2: Ezcaray, R-3: Santurde, R-4: Santo Domingo de la Calzada, R-5: Casalarreina. 
Tabla 1. Número de ejemplares de Hydropsychidae recogidos durante los años 2003-2005 en el río Oja (La Rioja, España). Puntos de muestreo: R-0-A: afluente del Oja, embalse del Águila, R-0: Oja, embalse del Águila, R-1: Oja-Arroyo Ortigal, R-2: Ezcaray, R-4: Santo Domingo de la Calzada, R-5: Casalarreina. Fechas de muestreo: 1: Mayo 2003, 2: Agosto 2003, 3: Noviembre 2003, 4: Febrero 2004, 5: Junio 2004, 6: Noviembre 2004, 7: Marzo 2005, 8: Mayo 2005, 9: Junio 2005, 10: Agosto 2005, 11: Junio 2006. Number of Hydropsychidae specimens collected during 2003-2005 in Oja River (La Rioja, Spain) Sampling points: R-0-A: Oja's tributary, Aguila's dam, R-0: Oja River, Aguila's dam, R-1: Oja River-Ortigal Brook, R-2: Ezcaray, R-4: Santo Domingo de la Calzada, R-5: Casalarreina. Sampling date: 1: May 2003, 2: August 2003, 3: November 2003, 4: February 2004, 5: June 2004, 6: November 2004, 7: March 2005, 8: May 2005, 9: June 2005, 10: August 2005, 11: June 2006.

\begin{tabular}{|c|c|c|c|c|c|c|c|c|c|c|c|c|c|c|c|c|c|c|c|c|c|}
\hline \multirow{2}{*}{$\begin{array}{l}\text { Punto de muestreo } \\
\text { Fecha }\end{array}$} & \multicolumn{2}{|c|}{ R-0-A } & \multicolumn{2}{|c|}{ R-0 } & \multicolumn{7}{|c|}{ R-1 } & \multicolumn{3}{|c|}{ R-2 } & \multicolumn{2}{|l|}{ R-4 } & \multicolumn{5}{|c|}{ R-5 } \\
\hline & 0 & 10 & 8 & & 1 & 3 & 7 & 5 & 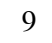 & 10 & & 1 & 2 & 3 & 3 & 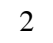 & 8 & 4 & 6 & 1 & 11 \\
\hline ydropsyche ambig & & . & & T & 10 & $J$ & & 40 & 1 & - & 20 & 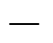 & & 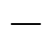 & 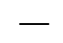 & & - & & - & - & - \\
\hline$y d r$ & - & - & - & - & - & - & - & - & - & - & - & - & - & - & - & - & 4 & - & 10 & - & - \\
\hline$r a$ & 19 & 4 & 5 & - & - & 1 & 27 & 1 & - & - & - & - & 4 & - & - & - & - & - & - & - & - \\
\hline Hyd & 2 & 12 & - & 1 & 3 & 1 & 1 & 3 & 2 & - & 7 & 1 & - & - & - & - & - & - & - & - & - \\
\hline Hyd & - & - & - & - & - & - & - & - & - & - & - & - & 2 & - & 1 & 64 & 287 & 21 & 133 & 3 & - \\
\hline Hydr & - & - & - & - & - & - & - & - & - & - & - & - & - & - & - & 3 & 13 & 3 & 26 & - & 3 \\
\hline ggnita & - & - & - & - & - & - & - & - & - & - & - & - & - & - & - & 3 & 38 & 1 & 43 & 1 & 3 \\
\hline tabilis & - & - & - & - & - & - & - & - & - & - & - & 1 & - & - & - & 2 & 1 & - & 1 & - & - \\
\hline Hyd & - & 1 & - & - & - & - & - & - & - & 1 & - & - & - & - & - & - & - & - & - & - & - \\
\hline Hydropsyche siltalai & - & - & - & - & - & - & - & - & - & - & - & 2 & 1 & 2 & - & - & 18 & 11 & 11 & - & 20 \\
\hline
\end{tabular}

por otra, en la aportación de posibles nuevas especies al listado de los Hydropsychidae ibéricos.

\section{ÁREA DE ESTUDIO}

El estudio se llevó a cabo en el río Oja (La Rioja, España) (Fig. 1). Nace en la Sierra de la Demanda $(1880 \mathrm{~m})$ y desemboca en el Tirón, poco antes de su desembocadura en el Ebro, estando su recorrido (54,5 Km.) circunscrito íntegramente a la Rioja Alta. De régimen pluvionival, se caracteriza por grandes variaciones de caudal entre distintas épocas del año, debido por una parte a la fuerte pendiente de su cauce, que hace que la escorrentía sea importante, y por otra, a la fuerte utilización del acuífero aluvial en agricultura intensiva, con importantes extracciones de agua en los momentos de menor caudal (verano).

Se seleccionaron cinco puntos de muestreo a lo largo del río, añadiéndose uno más en 2005, cercano a su nacimiento. Los puntos son: en cabecera, R-0 y R-0-A (1200 m, UTM 30T 493900 4673000), situados a la altura del Embalse del Águila, en el Oja y un afluente respectivamente, R-1 (944 m, UTM 30T 496378
4675906), donde se unen el Oja y el arroyo Ortigal. En el tramo medio-bajo: R-2 (719 m, UTM 30T 499284 4687396) en Ezcaray, por debajo de los efluentes de la depuradora; R-3 (711 m, UTM 30T 502037 4693135) en Santurde, a la entrada del valle aluvial; R-4 (616 m, UTM 30T 503531 4702049) en Santo Domingo de la Calzada, en plena zona de regadío y R-5 (569 m, UTM 30T 5070014710589 ) en Casalarreina, poco antes de su unión con el río Tirón. Exceptuando R-2, que se sitúa por debajo de una depuradora, en el resto de los puntos seleccionados no hay constancia de efluentes de poblaciones al río, aunque probablemente haya vertidos poco antes de R-5, procedentes de un camping y una piscifactoría próximos al río.

\section{MATERIAL Y MÉTODOS}

Durante un período anual (2003-2004) se tomaron muestras cuantitativas de macroinvertebrados en los cinco puntos citados (Mayo, R-1: 6 muestras, R-2: 12 m., R-3: 12 m., R-4: 8 m., R-5: 6 m.; Agosto, R-1: 6 m., R-2: 6 m., R-5: 6 m; Noviembre, R-1: 2 m., R-2: 6 m., R-3: 6 m., R-4: 6 m., R-5: 6 m.; Febrero, R-1: 3 m., 
R-2: 3 m., R-3: 3 m., R-4: 3 m., R-5: 3 m.), que se completaron con muestras cualitativas puntuales en R-1 (Junio 2004, Junio 2005, Agosto 2005, Junio 2006), R-5 (Noviembre 2004, Marzo 2005, Junio 2006), R-0 y R-0-A (Mayo 2005, Agosto 2005). Se utilizó un dispositivo tipo surber $30 \times 30 \mathrm{~cm}$., con una manga de nytal de 250 $\mu \mathrm{m}$ de luz de malla. Al mismo tiempo, en el momento de tomar las muestras cuantitativas se colocaron aguas arriba redes de deriva de $30 \times$ $30 \mathrm{~cm}$., $250 \mu \mathrm{m}$ de luz de malla, durante 30 minutos. Las muestras se fijaron con formol al $4 \%$ tamponado con $\mathrm{CINa}$ al $0.9 \%$. En el laboratorio los organismos se separaron con una lupa binocular y se determinaron con la máxima resolución taxonómica posible, conservándose posteriormente en alcohol de 70. Los recuentos presentados incluyen todos los organismos recogidos (bentos y derivas) en cada punto.

Para la determinación se utilizaron tanto claves generales (Edington \& Hildrew,1981; Zamora-Muñoz et al., 1995; Waringer \& Graf, 1997; Vieira-Lanero, 2000; Malicky, 2004), como descripciones de especies (Philipson, 1953; Coineau \& Jacquemart, 1963; Szczesny, 1974; Verneaux \& Faessel, 1976; García De Jalón, 1981; Bournaud, et al., 1982; García de Jalón, 1983; González \& Botosaneanu 1985; Pitsch, 1993; Vieira-Lanero et al., 2001, Zamora-Muñoz, 2002). Por último, las especies dudosas se consultaron con expertos. La distribución se obtuvo de García de Jalón (1982 b) y González et al. (1992).

\section{RESULTADOS}

\section{Faunística}

Se recolectaron un total de 3758 individuos (2728 larvas y 1030 pupas), pertenecientes a 49 especies (16 familias) de Tricópteros presentes en la Península Ibérica (el listado completo se publicará próximamente). La Tabla 1 recoge los individuos (873 larvas y 90 pupas en distinto grado de maduración) que pertenecen a la familia Hydropsychidae, género Hydropsyche Pictet, 1834 (L: larvas, P: pupas, o macho, o hembra e I indeterminadas):
Hydropsyche ambigua Schmid en Botosaneanu \& Schmid, 1973. (95 L, $21 \mathrm{P} \mathrm{o}$, $23 \mathrm{P}$ \%, 6 PI). Especie endémica de la Península Ibérica, se localiza en las cuencas del norte y del centro. Nueva cita para La Rioja.

Hydropsyche angustipennis (Curtis, 1834)(14 L). Especie europea, se distribuye también por Anatolia. En España se ha encontrado hasta la fecha únicamente la subespecie $H$. angustipennis mallorcana, aunque algunos expertos (ZamoraMuñoz, comunicación personal) la han encontrado en Cataluña. Primera cita para la Península Ibérica.

Hydropsyche bulbifera McLachlan, 1878 (61 L). Se distribuye por Europa Central y meridional y Anatolia. En la Península presenta una amplia distribución. Nueva cita para La Rioja.

Hydropsyche dinarica Marinkovic-Gospodnetic, 1979 (14 L, 4 P ơ, 2 P o, 3 PI). Especie de Europa central y Mediterráneo occidental, en la Península presenta una amplia distribución. Nueva cita para La Rioja.

Hydropsyche exocellata Dufour, 1841 (505 L, $4 \mathrm{P} \sigma^{\top}, 2 \mathrm{P}$ o). Especie de Europa occidental, con amplia distribución en la Península y nueva cita para La Rioja. Es la especie más abundante

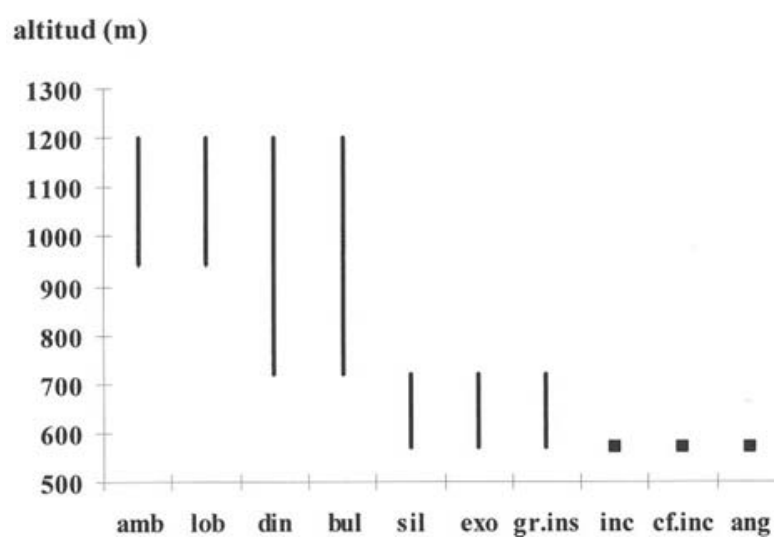

Figura 2. Distribución altitudinal de las especies; amb: Hydropsyche ambigua, lob: $H$. lobata, din: $H$. dinarica, bul: $H$. bulbifera, sil: $H$. siltalai, exo: $H$. exocellata, gr. ins: $H$. grupo instabilis, inc: $H$. incognita, cf. inc: $H$. cf. incognita, ang: $H$. angustipennis. Altitudinal distribution of species; $\mathbf{a m b}$ : Hydropsyche ambigua, lob: H. lobata, din: H. dinarica, bul: H. bulbifera, sil: H. siltalai, exo: H. exocellata, gr. ins: H. grupo instabilis, inc: H. incognita, $\boldsymbol{c f}$. inc: $\mathrm{H}$. cf. incognita, ang: $\mathrm{H}$. angustipennis. 
en este río, tanto dentro de los Hydropsychidae como del total de Tricópteros encontrados.

Hydropsyche gr. instabilis, (5 L). En una publicación anterior (Valladolid et al., 2006) aparece citada como $H$. fontinalis. Zamora-Muñoz (comunicación personal) nos confirmó la similitud con otras larvas que habían encontrado en cuencas del Mediterráneo (Bonada et al., 2004), pertenecientes al grupo instabilis y pendientes de denominación.

Hydropsyche incognita Pitsch, 1993 (46 L, 2 PI). Según una revisión de Malicky, en la Península Ibérica aparece citada numerosas veces como $H$. pellucidula (Vieira-Lanero, 2000). Navás (1908) y García de Jalón (1982b) la citan en La Rioja. Especie distribuida por Europa, norte de África y Asia suroccidental, en la Península presenta una amplia distribución.

Hydropsyche cf. incognita (86 L, 3 P o). Morfológicamente se parece mucho a $H$. incognita, sobre todo a nivel cefálico y de prosternitos, pero se diferencia claramente en los escleritos torácicos dorsales. Posible nueva especie.

Hydropsyche lobata McLachlan, 1884 (2 L). Especie presente en la Península Ibérica y norte de África, de amplia distribución, es nueva cita para La Rioja.

Hydropsyche siltalai Dohler, 1963 (45 L, 1 P ơ, 15 P o, 4 PI). Especie de Europa y Anatolia, de amplia distribución en la Península, aparece citada para La Rioja por García de Jalón (1982 b).

\section{Distribución longitudinal y estacional}

El río Oja presenta dos tramos definidos según sus características físicas: 1. Zona de cabecera, con fondos de grandes bloques y cantos de gran tamaño (20-40 cm), un cauce entre 3-5 m, altitud entre $2000-900 \mathrm{~m}$, y una pendiente del $12 \%$, que se correspondería aproximadamente con el ritron definido por García de Jalón (1986) para los ríos de la cuenca del Duero. 2. Zona medio-baja, con

Tabla 2. Comparación del número de especies de Hydropsychidae encontrados en el Oja y en otros ríos citados en la literatura. Cursiva: ríos europeos, $N^{o}$ est. $m$.: número de estaciones de muestreo en $c p$ : cauce principal, $a$ : afluentes, $N^{o} s p .:$ número de especies, $T$ : totales, $(c p)$ : especies encontradas en el cauce principal, Altitud: entre paréntesis, cotas máxima y mínima estimadas (no citadas en el texto). Comparison between the number of Hydropsychidae species found in Oja River and other rivers quoted in the literature. Italics: European rivers, $N^{o}$ est. m.: number of sampling stations in cp: main channel, a: tributaries, $\mathrm{N}^{\mathrm{o}}$ sp.: number of species, T: total number, (cp): species from main channel, Altitude: in brackets, estimated maximum and minimum height (not cited in text).

\begin{tabular}{|c|c|c|c|c|}
\hline Río (Localidad) & $\mathbf{N}^{0}$ est. $\mathbf{m} .(\mathbf{c p}+\mathbf{a})$ & $\mathbf{N}^{0}$ sp. $\mathbf{T}(\mathbf{c p})$ & Altitud & Referencia bibliográfica \\
\hline Usk (Reino Unido) & 45 & $6(4)$ & & Hildrew \& Edington (1979) \\
\hline Ródano (Francia) & $14(13 c p+1 a)$ & $7(7)$ & $(590-10 \mathrm{~m})$ & Bournaud et al. (1982) \\
\hline Lozoya (Madrid) & $12(9 \mathrm{cp}+3 \mathrm{a})$ & $7(6)$ & $(370-165 \mathrm{~m})$ & García de Jalón (1982) \\
\hline Alto Tajo (Guadalajara-Cuenca-Teruel) & $27(13 \mathrm{cp}+14 \mathrm{a})$ & $6(5)$ & $1608-1030 \mathrm{~m}$ & Herranz \& $\mathrm{G}^{\mathrm{a}}$ de Jalón (1984) \\
\hline Duero (Castilla-León) & $74(14 c p+60 a)$ & $10(8)$ & $1560-730 \mathrm{~m}$ & García de Jalón (1986) \\
\hline Viao-Piloña (Asturias) & $48(30 \mathrm{cp}+18 \mathrm{a})$ & $3(3)$ & $1300-190 \mathrm{~m}$ & Miranda Braga (1987) \\
\hline Ter (Gerona) & $20(12 \mathrm{cp}+8 \mathrm{a})$ & $7(4)$ & $440-30 \mathrm{~m}$ & Puig et al. (1987) \\
\hline Lea (Vizcaya) & $10(5 \mathrm{cp}+5 a)$ & $6(6)$ & $1300-20 \mathrm{~m}$ & Basaguren (1990) \\
\hline P.N. Los Alcornocales (Cádiz) & 30 (7 cuencas) & 4 & $210-20 \mathrm{~m}$ & Ruiz et al. (2001) \\
\hline Ter (Gerona) & $25(5 c p+20 a)$ & $3(1)$ & $1440-640 \mathrm{~m}$ & Bonada et al. (2004) \\
\hline Llobregat (Barcelona) & $25(13 \mathrm{cp}+12 \mathrm{a})$ & $8(5)$ & $1360-20 \mathrm{~m}$ & Bonada et al. (2004) \\
\hline Mijares (Teruel-Castellón) & $9(5 c p+4 a)$ & $5(5)$ & $1370-90 \mathrm{~m}$ & Bonada et al. (2004) \\
\hline Turia (Teruel-Cuenca-Valencia) & $13(8 \mathrm{cp}+5 \mathrm{a})$ & $3(3)$ & $1530-95 \mathrm{~m}$ & Bonada et al. (2004) \\
\hline Júcar (Teruel-Albacete-Cuenca-Valencia) & $17(7 \mathrm{cp}+10 \mathrm{a})$ & $6(6)$ & $1300-125 \mathrm{~m}$ & Bonada et al. (2004) \\
\hline Oja (La Rioja) & $7(6 \mathrm{cp}+1 \mathrm{a})$ & $10(10)$ & $1200-569 \mathrm{~m}$ & Estudio actual \\
\hline
\end{tabular}


cantos rodados de tamaño medio-pequeño (10$20 \mathrm{~cm}$ ) y numerosas zonas de arena, altitud entre 900- $475 \mathrm{~m}$ y una pendiente menor del $6 \%$, que se correspondería con el potamon definido por García de Jalón (1986).

Según su distribución a lo largo del eje del río (Fig. 2) podemos distinguir dos grupos de especies. El primero, que podría definirse como pertenecientes al ritron, está constituido por: Hydropsyche ambigua, H. lobata, $H$. bulbifera y $H$. dinarica. Están presentes en las zonas de cabecera del río (R-0 y R-1), presentándose las dos últimas también en la zona media (R2, $700 \mathrm{~m}$ ). En los dos muestreos realizados en R-0 la especie más abundante es $H$. ambigua, mientras que en R-0-A, $H$. ambigua es la más abundante en mayo, siendo sustituida en agosto por $H$. bulbifera. La diferente composición en especies en este punto, donde se unen el río Oja y un afluente, es debida probablemente a las diferencias en el sustrato: grandes rocas en el Oja, con pozas y zonas de corriente, mientras que en el afluente encontramos un fondo pedregoso con cantos de diverso tamaño y una lámina de agua de poca profundidad.

En el punto R-1 también aparece $H$. ambigua como especie dominante en mayo y noviembre de 2003 y junio de 2004, mientras que en febrero de 2004 la especie mayoritaria es $H$. bulbifera. Se observa que en los meses de verano solo aparecen larvas de los primeros estadíos. En este período las condiciones del río empeoran, con un descenso del caudal y un aumento de la temperatura del agua. Por el contrario, en agosto de 2005 en R-0 y R-0-A se siguen encontrando larvas de las especies presentes en otros meses, por lo que suponemos que su ciclo vital se alarga un poco más que en R-1. Por último, comentar que en agosto de 2005 aparecieron las larvas de $H$. lobata, tanto en R-0-A como en $\mathrm{R}-1$. En una publicación anterior (Valladolid $e t$ $a l ., 2006)$ citábamos a $H$. lobata como especie presente, aunque comprobaciones posteriores a la publicación indicaron que se trataba de $H$. ambigua. Los nuevos ejemplares obtenidos nos permiten confirmar la presencia de esta especie.

El segundo grupo de especies, que aparece en los tramos medio-bajos del río y que formarían parte del potamon lo compone: Hydropsyche angustipennis, $H$. incognita, $H$. cf. incognita, $H$. exocellata, $H$. gr. instabilis, y $H$. siltalai. Se han encontrado en el punto R-5 (569 m) y de éstas, las tres últimas aparecen también en el punto R2. Suponemos que también aparecerán en puntos intermedios (R-3 y R-4), aunque debido a las sequías periódicas que sufre el río, en estas zonas no se han capturado ejemplares.

De este grupo, la especie más abundante es H. exocellata, siendo también la más abundante del total de especies (Tabla 1). Hemos encontrado
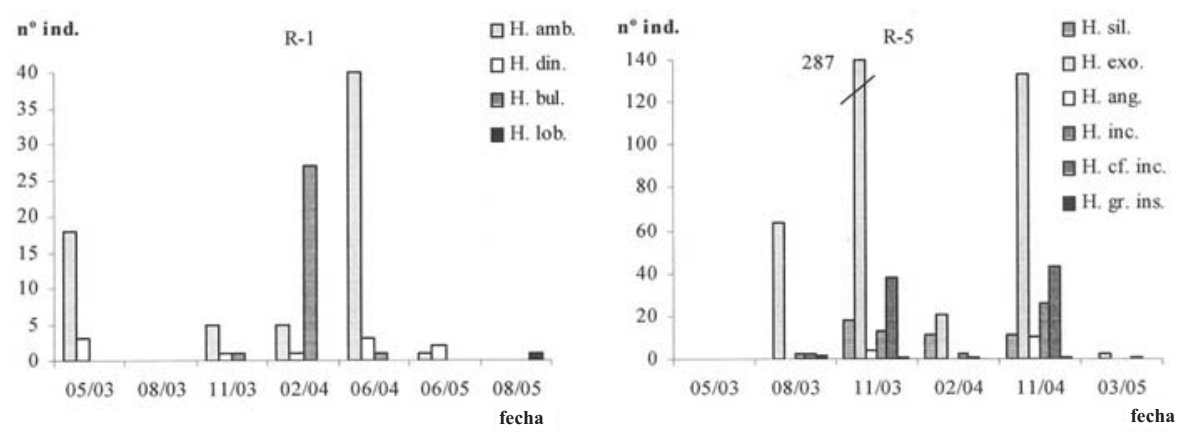

Figura 3. Distribución estacional de las distintas especies en cabecera (R-1: Oja-Arroyo Ortigal) y en el tramo inferior (R-5: Casalarreina). Fecha: $\mathrm{mm} / \mathrm{aa}, \mathbf{n}^{\mathbf{0}}$ ind: número de individuos recogidos. Especies: H. amb: Hydropsyche ambigua, H. din: $H$. dinarica, H. bul: H. bulbifera, H. lob: H. lobata, H. sil: H. siltalai, H. exo: H. exocellata, H. ang: H. angustipennis, H. inc: H. incognita, H. cf. inc: $H$. cf. incognita, H. gr. ins: $H$. instabilis gr. Seasonal distribution of species in headwaters (R-1: Oja-Arroyo Ortigal) and downstream (R-5: Casalarreina). Date: $\mathrm{mm} / y y, \boldsymbol{n}^{\boldsymbol{o}}$ ind: number of individuals collected. Species: $\boldsymbol{H}$. $\boldsymbol{a m b}$ : Hydropsyche ambigua, $\boldsymbol{H}$. din: H. dinarica, $\boldsymbol{H}$. bul: H. bulbifera, H. lob: H. lobata, H. sil: H. siltalai, H. exo: H. exocellata, H. ang: H. angustipennis, H. inc: H. incognita, H. $\boldsymbol{c f}$. inc: H. cf. incognita, H. gr. ins: H. instabilis $g r$. 
larvas de diferentes estadíos a lo largo de todo el año, lo que indica que se producen varias generaciones anuales de adultos (Vieira-Lanero, 2000). Le siguen en abundancia $H$. siltalai y $H$. angustipennis, de esta última solo hemos colectado larvas de pequeño tamaño. Para finalizar, dos especies muy relacionadas, $H$. incognita, citada numerosas veces en la bibliografía como $H$. pellucidula, y otra especie que hemos denominado H. $c f$. incognita, debido al gran parecido con la anterior, aunque presenta diferencias en ciertas estructuras no utilizadas en la determinación de la especie, como p.e. los escleritos dorsales, por lo que suponemos que se trata de un taxón diferente, pendiente de comprobar.

Por último, comentar que el punto R-2 (Ezcaray), incluido por sus características en el tramo medio-bajo, aparece como zona fronteriza entre ambos ambientes, presentando especies de uno $\mathrm{u}$ otro grupo según las condiciones ambientales (Fig. 2): en mayo encontramos $H$. dinarica (ritron) junto con $H$. siltalai y $H$. $g r$. instabilis, (potamon) mientras que en agosto aparece $H$. bulbifera (ritron) junto con $H$. siltalai y $H$. exocellata, especie típica del potamon. Este fenómeno, que ha sido citado en la literatura, estaría asociado a la modificación de las condiciones ambientales, que adquieren carácter de potamon por la sequía, permitiendo la aparición de especies típicas de este medio durante algunos meses del año, mientras que en los meses de más caudal aparecen especies del ritron por arrastre (García de Jalón, 1986).

Únicamente en R-1 y en R-5 se han obtenido ejemplares en la mayoría de las campañas realizadas, por lo que restringimos el estudio de la distribución estacional a estos dos puntos (Fig. 3). Mientras que en R-1 se observa que el máximo de individuos de cada especie aparece en meses diferentes, en R-5 las abundancias máximas de todas las especies coinciden en el mes de noviembre, tanto en 2003 como en 2004.

\section{DISCUSIÓN}

Los Hydropsychidae constituyen un elemento importante en las comunidades de macroinver- tebrados acuáticos de todo tipo de ríos, ocupando desde la cabecera hasta los tramos más bajos (Verneaux \& Faesel, 1976; García de Jalón, 1986). En la Península Ibérica se ha confirmado la presencia de 24 especies de la familia, de las cuales 2 son del género Diplectrona, $1 \mathrm{del}$ género Cheumatopsyche y 21 del género $H y$ dropsyche (véase González, 2003). Si observamos el número medio de especies de Hydropsychidae que aparecen en diversos estudios sobre ríos europeos y españoles (Tabla 2), vemos que oscila entre 3 y 10 , aumentando a medida que aumenta el tamaño del río estudiado, y por tanto su cuenca. En el río Oja hemos encontrado un total de 10 especies, por lo que presenta una gran riqueza faunística en este aspecto (50\% de las especies de Hydropsyche presentes en la Península), teniendo en cuenta su longitud $(54.5 \mathrm{~km}$.) y su desnivel máximo $(700 \mathrm{~m})$. $\mathrm{Si}$ a esto añadimos el reducido número de localidades que se han muestreado, podemos concluir que el potencial en cuanto al número presente de especies puede ser todavía mayor, ya que, por las características del proyecto en que se engloba el estudio no se han muestreado afluentes, que podrían haber aportado especies no presentes en el cauce principal, como se observa en otros ríos (p.e., Hildrew \& Edington 1979; García de Jalón 1982 a, 1986; Herranz \& García de Jalón 1984; Puig et al. 1987; Bonada et al., 2004).

La distribución altitudinal de especies del ritron como $H$. dinarica y $H$. ambigua coincide con las citadas para otros ríos de la Península, mientras que otras como $H$. lobata o $H$. bulbifera se han citado en entornos diferentes. Puig et al. (1987) encuentran la especie $H$. c f. lobata en el río Rigart $(1100 \mathrm{~m})$, mientras que García de Jalón (1986) cita esta especie como habitante del epipotamon, en ríos pequeños de la cuenca del Duero, a altitudes entre 190-1080 m. Por otro lado, $H$. bulbifera aparece citada como especie de potamon, de tramos medios y bajos (García de Jalón 1982 b, 1986; Basaguren 1990, Bonada et al. 2004) . En nuestro caso, ambas especies se han encontrado siempre por encima de los $900 \mathrm{~m}$, en la zona que consideramos de ritron.

Respecto a las especies que encontramos en el potamon, $H$. siltalai se considera en Europa 
una especie de pequeños cursos de agua (Hildrew \& Edington 1979; Bournaud et al. 1982) y en la Península habita ríos de cabecera con fuerte corriente (Herranz \& García de Jalón 1984; García de Jalón 1986) . Nosotros la hemos encontrado en el tramo inferior, llegando hasta la zona intermedia de Ezcaray, por lo que la consideramos típica del potamon en este río.

En cuanto al resto de las especies del potamon, $H$. exocellata se ha citado en tramos inferiores de los ríos (Décamps 1968; Bournaud et al., 1982; García de Jalón, 1982 b, 1986; Puig et al., 1987; Basaguren 1990; Bonada et al., 2004), a menudo con una importante carga orgánica, mientras que $H$. angustipennis aparece en tramos medios y bajos, asociada a temperaturas más altas (Philipson \& Moorhouse 1974; Szczesny 1974). En cuanto a $H$. incognita, Malicky considera que en la Península Ibérica tradicionalmente se han determinado como $H$. pellucidula individuos que probablemente sean de esta especie (Vieira-Lanero, 2000), por lo que no tenemos elementos para comparar su distribución. En la literatura, tanto en ríos peninsulares como europeos, $H$. pellucidula presenta una distribución altitudinal y geográfica amplia (Philipson, 1957; Décamps, 1968; Edington \& Hildrew, 1973; Verneaux \& Faessel, 1976; Boon, 1979; Bournaud et al., 1982; García de Jalón, 1982 a, b, 1986; Basaguren, 1990) y suele aparecer asociado a $H$. siltalai, ocupando ésta última especie zonas de mayor corriente.

La distribución estacional de las especies solo se ha podido observar en los puntos R-1 y R-5, que son los que más número de muestras han aportado. Se observa que mientras en cabecera (R-1), cada especie tiene un máximo de abundancia en un mes concreto, en R-5 los máximos aparecen en noviembre, cuando aparecen además ejemplares de todas las especies. Estas diferencias se podrían explicar por las características del medio. En cabecera los recursos son más escasos, por lo que las especies deben repartirlos, bien con pequeñas diferencias en cuanto a preferencia de microhábitat, o bien modificando su ciclo vital, de manera que no coincidan en el tiempo larvas de tamaños similares. Estudios europeos realizados con especies que conviven en el mismo tramo de río al mismo tiempo (Boon 1979; Hildrew \& Edington 1979), indican que hay diferencias específicas de tamaño de las larvas invernantes, que se corresponden con diferentes tipos de alimentación (en cuanto a tamaño y clase de partículas ingeridas) y preferencias de microhábitat. En ambos casos se observaba que $H$. siltalai y $H$. pellucidula presentaban una tasa de crecimiento diferente, coincidiendo en el tiempo larvas en distintas etapas de desarrollo y, por tanto, con dietas diferentes. Además, las larvas de $H$. siltalai, de menor tamaño, tenían preferencia por la zona superior de las piedras, cubiertas de un musgo de crecimiento estacional y expuestas a una mayor velocidad de la corriente, mientras que las larvas de $H$. pellucidula ocupaban los lugares debajo de las piedras, con menos corriente.

Por otro lado, en los tramos bajos la cantidad de alimento disponible se aumenta considerablemente, tanto a nivel de materia orgánica como de biomasa, incrementándose el número de nichos, (Margalef, 1960) lo que permite la coexistencia de especies con requerimientos parecidos en la misma época del año.

Podemos concluir diciendo que la combinación de diferentes velocidades de desarrollo larvario, tipos de alimento y microhábitats preferidos permite a las distintas especies una distribución espacial y temporal única, evitando o minimizando la competencia directa.

\section{AGRADECIMIENTOS}

Este trabajo de investigación se ha financiando a través del Proyecto del Plan Nacional de I+D (REN2002-02550). Ha contado con el apoyo de la Unión de Agricultores y GanaderosCoordinadora de Agricultores y Ganaderos (UAGR-COAG) mediante un Contrato Tecnológico (Ref. 2003/672). La Consejería de Educación de la Comunidad de Madrid y el Fondo Social Europeo (F.S.E.) participan mediante la financiación de una beca de Formación de Personal Investigador. Queremos agradecer también a Carmen Zamora-Muñoz, Marcos González, Bronislaw Szczesny y Peter Neu su ayuda en la determinación, tanto aportando bibliografía como 
ejemplares y comprobando directamente alguna de las especies encontradas.

\section{BIBLIOGRAFÍA}

BASAGUREN, A. 1990. Distribución de las especies pertenecientes a la familia Hydropsychidae (Trichoptera) en la cuenca del Lea (País Vasco). Scientia gerundensis, 16(1): 43-52.

BONADA, N., C. ZAMORA-MUÑOZ, M. RIERADEVALL \& N. PRAT. 2004. Trichoptera (Insecta) collected in Mediterranean River Basins of the Iberian Peninsula: taxonomic remarks and notes on ecology. Graellsia, 60(1): 41-69.

BOON, P. J. 1979. Studies on the spatial and temporal distribution of larval Hydropsychidae in the North Tyne river system (Northern England). Arch. Hydrobiol., 85(3): 336-359.

BOURNAUD, M., H. TACHET, et J. F. PERRIN. 1982. Les Hydropsychidae (Trichoptera) du HautRhône entre Genève et Lyon. Annls. Limnol., 18(1): 61-80.

COINEAU, Y. et S. JACQUEMART. 1963. Missions S. Jacquemart dans les Pyrénées Orientales (Quatrième note). A propos de quelques Trichoptères des Pyrénées Orientales. Bull. Inst. R. Sci. Nat. Belgique, 39 (6): 1-40

DÉCAMPS, H. 1968. Vicariances écologiques chez les Trichoptères des Pyrénées. Annls. Limnol., 4(1): 1-50.

EDINGTON, J. M. \& A. G. HILDREW. 1973. Experimental observations relating to the distribution of net-spinning Trichoptera in streams. Verh. int. Verein. Limnol., 18: 1549-1558.

EDINGTON, J. M. \& A. G. HILDREW. 1981. Caseless caddis larvae of the British Isles. Freshwater Biological Association. Scientific Publication 43, $91 \mathrm{pp}$.

GARCÍA DE JALÓN, D. 1981. Description of Hydropsyche larvae found in the Iberian Peninsula. In: Proc. of the 3rd Int. Symp. on Trichoptera. G. P. Moretti (ed.): 87-92. Junk Publishers, The Hague.

GARCÍA DE JALÓN, D. 1982a. Los Trichoptera del río Lozoya. Bol. Asoc. esp. Entom., 5: 41-58

GARCÍA DE JALÓN, D. 1982b. Contribución a la zoogeografía de los Tricópteros españoles (II). SHILAP, 10: 157-165.

GARCÍA DE JALÓN, D. 1983. Contribución al conocimiento de las larvas del género Hydropsyche
(Trichoptera) ibéricas. Actas I Congreso Iberico de Entomologia, 1(1): 275-285.

GARCÍA DE JALÓN, D. 1986. Los Hydropsychidae (Trichoptera) de la Cuenca del Duero. Bol. Asoc. esp. Entom., 10: 127-138.

GIUDICELLI, J. M., M. DAKKI et A. DIA. 1985. Characteristiques abiotiques et hydrobiologiques des eaux courantes mediterranéennes. Verh. int. Verein. Limnol., 22: 2094-2101.

GONZÁLEZ, M. 2003. El Reino Animal en la Península Ibérica y las Islas Baleares. O. Trichoptera, Fam. Hydropsychidae Curtis, 1835. http: //www.fauna-iberica.mncn.csic.es/faunaib/arthropoda/insecta/trichoptera/hydropsychidae.php

GONZÁLEZ, M. A. \& L. BOTOSANEANU. 1985. Étude de trois espèces de Hydropsyche Pictet d'Espagne décrites par F. Schmid (Insecta: Trichoptera). Bull. zool. Mus. Univ. Amsterdam, 10 (13): 89-95.

GONZÁLEZ, M. A. \& H. MALICKY. 1999. Une nouvelle espèce de Hydropsyche du groupe pellucidula (Trichoptera, Hydropsychidae). Braueria, 26: 25-26.

GONZÁLEZ, M. A., L. S. W. TERRA, D. GARCÍA DE JALÓN y F. COBO. 1992. Lista faunística y bibliográfica de los Tricópteros (Trichoptera) de la Península Ibérica e Islas Baleares. Asoc. esp. Limnol., Publ. N ${ }^{\circ} 11.200$ pp.

HERRANZ, J. M. y D. GARCÍA DE JALÓN, D. 1984. Distribución de las especies del género Hydropsyche (O. Trichoptera, Hydropsychidae) en la cuenca del Alto Tajo (Guadalajara). Limnetica, 1: 203-206.

HILDREW, A. G. \& J. M. EDINGTON. 1979. Factors facilitating the coexistence of Hydropsychid caddis larvae (Trichoptera) in the same river system. J. Anim. Ecol., 48: 557-576.

MALICKY, H. 2004. Atlas of European Trichoptera. 2nd Edition. Springer-Verlag, 359 pp.

MARGALEF, R. 1960. Ideas for a synthetic approach to the ecology of running waters. Int. Rev. ges. Hydrobiol., 45(1): 133-153.

MILLET, X. 1983. Les larves del gènere Hydropsyche (Insecta: Trichoptera) a Catalunya. Taxonomia. Bull. Inst. Cat. Hist. Nat., 49 (Secc. Zool., 5): 97-103.

MIRANDA BRAGA, A. 1987. Utilización de macroinvertebrados bénticos como indicadores biológicos de la calidad del agua en el río Viao-Piloña (Asturias). Limnetica, 3: 141-150. 
NAVÁS, L. 1908. Neurópteros de España y Portugal. Broteria (Ser. Zool.), 7: 5-131.

PHILIPSON, G. N. 1953. The larva and pupa of Hydropsyche instabilis Curtis (Trichoptera, Hydropsychidae). Proc. R. Ent. Soc. London (A), 28 (1-3): 17-23.

PHILIPSON, G. N. 1957. Records of caddis-flies (Trichoptera) in Northumberland with notes on their seasonal distribution in Plessey Woods. Trans. Nat. Hist. Soc. Northumberland (New Series), XII: 77-92.

PHILIPSON, G. N. \& B. H. S. MOORHOUSE. 1974. Observations on ventilatory and net- spinning activities of the larvae of the genus Hydropsyche Pictet (Trichoptera: Hydropsychidae) under experimental conditions. Freshwat. Biol., 4: 525-533.

PITSCH, T. 1993. Zur kenntnis der Hydropsyche pellucidula-Grupe in Mitteleuropa (Trichoptera: Hydropsychidae). Braueria, 20: 27-32.

PUIG, M. A., G. GONZÁLEZ y O. SORIANO. 1984. Introducción al estudio de las comunidades macrobentónicas de los ríos asturianos: Efemerópteros, Plecópteros, Tricópteros, Simúlidos y Quironómidos. Limnetica, 1: 187-196.

PUIG, M. A., G. GONZÁLEZ y L. RECASENS. 1987. Modelos de distribución de plecópteros, efemerópteros, Tricópteros y simúlidos en el río Ter. Limnetica, 3: 125-132.

RUIZ, A., J. C. SALAMANCA-OCAÑA. y M. FERRERAS-ROMERO. 2001. Fauna de Tricópteros (Insecta:Trichoptera) de cursos de agua que drenan canutos del Parque Natural Los Alcornocales (sur de España). Boln. Asoc. esp. Ent., 25(3-4): 105-120.

SZCZESNY, B. 1974. Larve of the genus Hydropsy- che (Insecta, Trichoptera) from Poland. Pol. Arch. Hydrobiol., 21(3/4): 387-390.

VALLADOLID, M., J. J. MARTÍNEZ-BASTIDA, M. ARAUZO y C. GUTIÉRREZ. 2006. Abundancia y biodiversidad de los macroinvertebrados del río Oja (La Rioja, España). Limnetica, 25 (3-4): 133-140.

VERNEAUX, J. et B. FAESSEL. 1976. Larves du genre Hydropsyche (Trichoptères Hydropsychidae). Taxonomie, données biologiques et écologiques. Annls. Limnol., 12 (1): 7-16.

VIEIRA-LANERO, R. 2000. Las larvas de los Tricópteros de Galicia (Insecta: Trichoptera). Tesis Doctoral. Universidad de Santiago de Compostela. 612 pp.

VIEIRA-LANERO, R., M. A. GONZÁLEZ \& F. COBO. 2001. The larva of Hydropsyche urgorrii González \& Malicky, 1980. Spixiana, 24 (2): 141-146.

WARINGER, J. \& W. GRAF. 1997. Atlas der österreichischen Köcherfliegenlarven: unter Einschluss der angrenzenden Gebiete. Wien. Facultas Univ.-Verl. 286 pp.

ZAMORA-MUÑOZ, C., J. ALBA-TERCEDOR \& D. GARCÍA DE JALÓN. 1995. The larvae of the genus Hydropsyche (Hydropsychidae; Trichoptera) and key for identification of species of the Iberian Peninsula. Mitt. Schweiz. Entomol. Ges., 68: 189-210.

ZAMORA-MUÑOZ, C., M. A. GONZÁLEZ, J. PICAZO-MUÑOZ \& J. ALBA-TERCEDOR. 2002. Hydropsyche fontinalis, a new species of the instabilis-Group from the Iberian Peninsula (Trichoptera, Hydropsychidae). Aquatic Insects, 24 (3): 189-197. 